\title{
Geodesic Matching of Triangulated Surfaces
}

\author{
A. Ben Hamza ${ }^{\star}$ and Hamid Krim ${ }^{\diamond}$ \\ ${ }^{\star}$ Concordia Institute for Information Systems Engineering \\ Concordia University, Montréal, QC, H3G 1T7 \\ ${ }^{\diamond}$ Department of Electrical and Computer Engineering \\ North Carolina State University, Raleigh, NC 27695-7914
}

\begin{abstract}
Recognition of images and shapes has long been the central theme of computer vision. Its importance is increasing rapidly in the field of computer graphics and multimedia communication because it is difficult to process information efficiently without its recognition. In this paper, we propose a new approach for object matching based on a global geodesic measure. The key idea behind our methodology is to represent an object by a probabilistic shape descriptor that measures the global geodesic distance between two arbitrary points on the surface of an object. In contrast to the Euclidean distance which is more suitable for linear spaces, the geodesic distance has the advantage to be able to capture the intrinsic geometric structure of the data. The matching task therefore becomes a one-dimensional comparison problem between probability distributions which is clearly much simpler than comparing 3D structures. Object matching can then be carried out by an information-theoretic dissimilarity measure calculations between geodesic shape distributions, and is additionally computationally efficient and inexpensive.
\end{abstract}

\section{INTRODUCTION}

In recent years there has been an increasing interest in recognition of images and shapes. Its importance is also increasing rapidly in the field of computer graphics and multimedia communication because it is difficult to process information efficiently without its recognition. Three-dimensional (3D) objects consist of geometric and topological information, and their compact representation is an important step towards a variety of computer vision applications including indexing, retrieval, and matching in a database of 3D models. The latter will be the focus of this paper. There are basically two major steps in object matching: the first step involves finding a reliable and efficient shape representation/descriptor, and the second step is the design of an appropriate dissimilarity measure for object comparison between the shape representations.

Most three-dimensional shape matching techniques proposed in the literature of computer graphics, computer vision and computer-aided design are based on geometric representations which represent the features of an object in such a way that the shape dissimilarity problem reduces to the problem of comparing two such object representations. Feature-based methods require that features be extracted and described before two objects can be compared [1,2]. Among feature-based methods, one popular approach is graph matching, where two objects are represented by their graphs composed of vertices and edges. An efficient representation that captures the topological properties of 3D objects is the Reeb graph descriptor proposed by Shinagawa et al. [1,3]. The vertices of the Reeb graph are the singular points of a function defined on the underlying object $[1,3-5]$. These singularities are prominent landmarks and their detection, recognition, and classification is a crucial step in image processing and computer vision [4]. Such singularities carry important information for further operations, such as image registration, shape analysis, motion estimation, object recognition, and surface evolution [6-8].

An alternative to feature-based representations, called shape distribution, is developed by Osada et al [9]. The idea here is to represent an object by a global histogram based on the Euclidean distance defined on the surface of an object. The shape matching problem is then performed by computing a dissimilarity measure between the shape distributions of two arbitrary objects. This approach is computationally stable and relatively insensitive to noise. Because of unsuitability of the Euclidean distance when dealing with nonlinear manifolds, the shape distribution, however, does not capture the nonlinear geometric structure of the data.

Information-theoretic measures provide quantitative entropic divergences between two probability distributions. A common entropic dissimilarity measure is the Kulback-Liebler (or directed) divergence [10] which has been successfully used in many applications including indexing and image retrieval [11]. Another entropy-based measure is the Jensen-Shannon (JS) divergence which may be defined between an arbitrary number of probability distributions [12]. Due to this generalization, the JS divergence may be used as a coherence measure between any number of distributions and may be applied to a variety of image processing and computer vision applications including graph matching [13], image registration and segmentation [14,15], edge detection [16], and segmentation of DNA sequences into homogenous domains [17].

In this paper, we propose a shape signature called geodesic shape distribution that captures the intrinsic geometric structure of $3 \mathrm{D}$ objects. A preliminary work on this signature was presented in [18]. The proposed method is inspired by previous works on object matching and in particular the recent works of Hilaga et al [19] and Osada et al [9]. The shape distribution approach [9] is based on the Euclidean distance which is not suitable for capturing the nonlinear structure of 3D objects, whereas the method presented in [19] is a feature-based technique and requires the extraction of the singularities of the geodesic distance. These singularities, however, are not easy to extract/compute and most algorithms give erroneous results. On the other hand, our approach is based on the triangle mesh centroids which are the simplest features to compute and effectively characterize the global surface shape. The 
primary motivation of the proposed approach is to encode a 3D shape into a one-dimensional geodesic distribution function. This compact and computationally simple representation is based on a global geodesic distance defined on an object surface, and takes the form of a kernel density estimate. The geodesic distance overcomes the limitations of the Euclidean distance which by virtue of its linearity in nature cannot account for nonlinear structures in the underlying 3D object. To gain further insight into the geodesic shape distribution and its practicality in $3 \mathrm{D}$ computer imagery, some numerical experiments are provided to demonstrate the potential and the much improved performance of the proposed methodology in 3D object matching. This was carried out by computing the JS divergence between probabilistic shape distributions. The geodesic shape distribution may be used to facilitate representation, indexing, retrieval, and object matching in a database of 3D models. More importantly, the geodesic shape distribution provides a new way to look at the object matching problem by understanding the intrinsic geometry of the shape.

The rest of this paper is organized as follows. The next section is devoted to the problem formulation. Section III describes some of the related work to our proposed approach for $3 \mathrm{D}$ object matching. In Section IV, we describe the representation step of our proposed shape signature called geodesic shape distribution, and we discuss its main properties. In Section V, we present the Jensen-Shannon divergence measure and show its attractive properties as a dissimilarity measure between probability distributions. In Section VI, we provide numerical simulations to show the power of the proposed shape measure for object matching. And finally, Section VII provides some conclusions.

\section{PROBlem FORMULATION}

Three-dimensional objects are usually represented as polygonal or triangle meshes in computer graphics and geometricaided design. A triangle mesh $\mathbb{M}$ is a pair $\mathbb{M}=(\mathcal{V}, \mathcal{T})$, where $\mathcal{V}=\left\{\boldsymbol{v}_{1}, \ldots, \boldsymbol{v}_{m}\right\}$ is the set of vertices, and $\mathcal{T}=$ $\left\{T_{1}, \ldots, T_{n}\right\}$ is the set of triangles.

In scientific visualization and analysis, a triangle mesh is too large to be examined without simplification. One way to overcome this limitation is to represent a triangle mesh by its surface features that can easily be computed and can effectively characterize the global surface shape. The centroids of the set of triangles $\mathcal{T}$ are desirable features which may be computed efficiently and have a global significance for the surface shape representation as illustrated in Fig. 1. In addition, there is a well defined correspondence between the centroid and the region (triangle) from which it is computed as depicted in Fig. 1. It is important to point out that centroidbased methods have been used in a variety of computer vision applications including clustering, and one of the widely centroid-based technique used for cluster analysis in the $\mathrm{K}$ mean algorithm [20].

\section{A. Global shape measure}

Let $\mathbb{M}=(\mathcal{V}, \mathcal{T})$ be a triangle mesh. The centroid $c_{j}$ of a triangle $T_{j}$ is the mean of its vertices, that is, the point located at the center of the triangle. Note that the cardinality of the set of centroids $\mathcal{C}=\left\{\boldsymbol{c}_{\boldsymbol{1}}, \ldots, \boldsymbol{c}_{\boldsymbol{n}}\right\}$ of the triangle mesh $\mathbb{M}$ is equal to the cardinality of its set of triangles $\mathcal{T}$.

Unless we establish a meaningful measure of distance between the centroids of a triangle mesh, no meaningful exploration of the underlying structure of an object is possible. In order to take into account the interaction between the centroids, we compute a pairwise distance measure $d\left(\boldsymbol{c}_{i}, \boldsymbol{c}_{j}\right)$ from any centroid $\boldsymbol{c}_{i}$ to all the other centroids $\boldsymbol{c}_{j} \in \mathcal{C}$. Fig. 1 illustrates an arbitrary distance between two centroids. Notice that distance $d$ need not be a Euclidean metric.

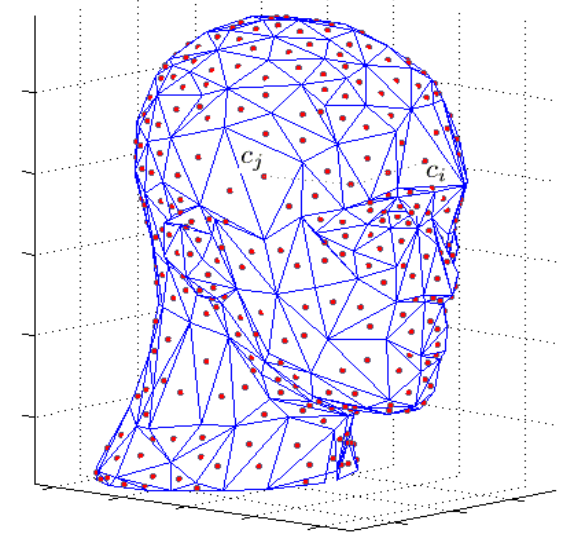

Fig. 1. Distance between two arbitrary centroids of a 3D object.

To obtain a global measure of the shape $\mathbb{M}$, we simply integrate (sum) over all centroids. More precisely, we define a function $f: \mathcal{C} \rightarrow \mathbb{R}$ such that

$$
f\left(\boldsymbol{c}_{i}\right)=\frac{1}{|\mathcal{C}|} \sum_{j=1}^{n} \operatorname{area}\left(T_{j}\right) d\left(\boldsymbol{c}_{i}, \boldsymbol{c}_{j}\right)^{2},
$$

where area $\left(T_{j}\right)$ denotes the area of the triangle $T_{j}$, and $|\mathcal{C}|=$ $\sum_{j=1}^{n} \operatorname{area}\left(T_{j}\right)$ is the total area of the surface $\mathbb{M}$. The function $f$ clearly represents a global measure or a distribution of the shape and therefore to each $3 \mathrm{D}$ model $\mathbb{M}$ we will assign its global measure $f$.

The problem addressed in this paper may now be concisely described by the following statement: Given two 3D objects $\mathbb{M}_{1}$ and $\mathbb{M}_{2}$ to be matched, find their global measures $f_{1}$ and $f_{2}$, and calculate how dissimilar these objects are based on a predefined dissimilarity measure $D\left(f_{1}, f_{2}\right)$. In other words, the dissimilarity between two objects measures "how different they are", and a smaller value of $D$ means that the two objects are more similar.

\section{RELATED WORK}

In this section, we will review two representative methods for object matching that are closely related to our proposed approach. We briefly show their mathematical foundations and algorithmic methodologies as well as their limitations.

\section{A. Reeb graph}

Morse theory explains the presence and the stability of critical points in terms of the topology of the underlying smooth 
manifold [21-23]. Topology is the property that determines which parts of the shape of objects are connected to which other parts [24-26], while geometry determines where, in a given coordinate system, each part is located [27]. The basic principle is that the topology of a manifold is very closely related to the critical points of a smooth function on that manifold. An interesting concept related to Morse theory and very useful to analyze a surface topology is the Reeb graph. The latter is defined as a quotient space $\mathbb{M} / \sim$ with an equivalence relation given by $\boldsymbol{p} \sim \boldsymbol{q}$ if and only if $h(\boldsymbol{p})=h(\boldsymbol{q})$ and $\boldsymbol{p}, \boldsymbol{q}$ belong to the same connected component of $h^{-1}(h(\boldsymbol{p}))$, where $h: \mathbb{M} \rightarrow \mathbb{R}$ is the height function such that $h(x, y, z)=z$ for all $(x, y, z) \in \mathbb{M}$. In particular, each connected component is represented by a point in the Reeb graph. By taking an appropriate number of crosssections and smooth interpolation between, Shinagawa et al. $[1,3]$ proposed a Reeb graph based approach or so-called homotopy model for object reconstruction. Reeb graph has a nice mathematical definition that makes it very attractive from a theoretical point of view. This representation, however, is not rotationally invariant. This limitation lead Hilaga et al to develop a geodesic-based Reeb graph technique [19]. In this approach a multiresolution Reeb graph is computed efficiently and a similarity distance is calculated to compare two Reeb graphs.

\section{B. Shape distribution}

Recently, Osada et al [9] proposed a shape distribution based approach for three-dimensional object matching. The key idea is to compute the Euclidean distance between all pairs of random points on the surface to obtain the so-called D2 shape histogram. Given a triangle $T_{j}=\left\{\boldsymbol{v}_{j 1}, \boldsymbol{v}_{j 2}, \boldsymbol{v}_{j 2}\right\}$, each random point is generated as

$$
\boldsymbol{p}_{j}=\left(1-\sqrt{r}_{1}\right) \boldsymbol{v}_{j 1}+\sqrt{r_{1}}\left(1-r_{1}\right) \boldsymbol{v}_{j 2}+\sqrt{r_{1}} r_{2} \boldsymbol{v}_{j 3},
$$

where $r_{1}$ and $r_{2}$ are pseudo-random numbers between zero and the total cumulative area. Then, the comparison of objects is carried out by computing a dissimilarity measure between their D2 shape distributions. Fig. 2 illustrates an ellipsoid and its D2 shape distribution. The main drawback of the shape distribution approach, which is based on the Euclidean distance, is its inability to capture the nonlinear structure of the data.

\section{Shape spectrum}

Shape spectrum was initially proposed for view grouping and matching of 3D free-form objects [28], and it is the histogram of the shape index operator which is defined at each point $\boldsymbol{p}$ of a surface $\mathbb{M}$ as

$$
\mathcal{S}(\boldsymbol{p})=\frac{1}{2}-\frac{1}{\pi} \tan ^{-1} \frac{\kappa_{1}(\boldsymbol{p})+\kappa_{2}(\boldsymbol{p})}{\kappa_{1}(\boldsymbol{p})-\kappa_{2}(\boldsymbol{p})},
$$

where $\kappa_{1}, \kappa_{2}$ are the surface principal curvatures such that $\kappa_{1} \geq \kappa_{2}$. These principal curvatures measure the maximum and minimum bending of the surface at each point [27].

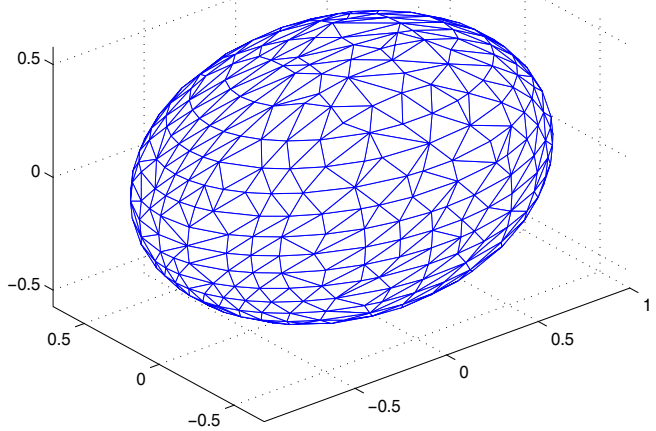

(a) Ellipsoid

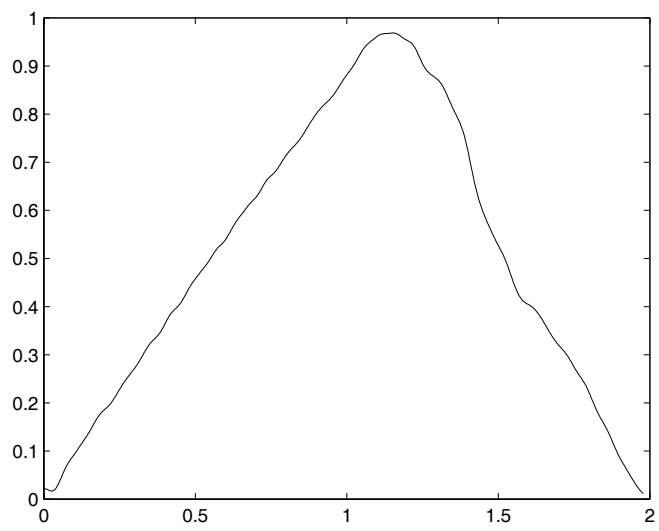

(b) D2 shape distribution

Fig. 2. D2 shape distribution of an ellipsoid. The horizontal axis in (b) represents the Euclidean distance, and the vertical axis represents the probability of that distance between two centroids on the surface.

Given a triangle mesh $\mathbb{M}=(\mathcal{V}, \mathcal{T})$, the principal curvatures at each face (or triangle) $T_{i} \in \mathcal{T}$ are computed by fitting a quadric surface $\mathbb{Q}=\{(x, y, u(x, y))\}$ where

$$
\left.u(x, y)=\lambda_{1} x^{2}+\lambda_{2} x y+\lambda_{3} y^{2}+\lambda_{4} x+\lambda_{5} y+\lambda_{6}\right\}
$$

to the set of points consisting of the centroid $c_{i}$ of $T_{i}$ and the centroids of the adjacent triangles to $T_{i}$ (i.e. the triangles that share an edge with $T_{i}$ ). Fig. 3 depicts a 3D object and its shape spectrum.

\section{PROPOSED APPROACH}

The goal of our proposed approach may be described as follows: Given two 3D objects $\mathbb{M}_{1}$ and $\mathbb{M}_{2}$ to be matched, find their global measures or shape descriptors $f_{1}$ and $f_{2}$, and calculate how dissimilar these objects using a dissimilarity measure $D\left(f_{1}, f_{2}\right)$ that has to be quantified. The basic idea behind the shape descriptor is to characterize a 3D object with a one-dimensional function which will help us discriminate between objects in a database of 3D models.

\section{A. Global geodesic shape function}

The Reeb graph concept has been shown to be very effective in modeling 3D objects based on cross sections such as MRI or CT images. It is more appropriate to modeling applications where the height is of special interest such as terrain imaging. 

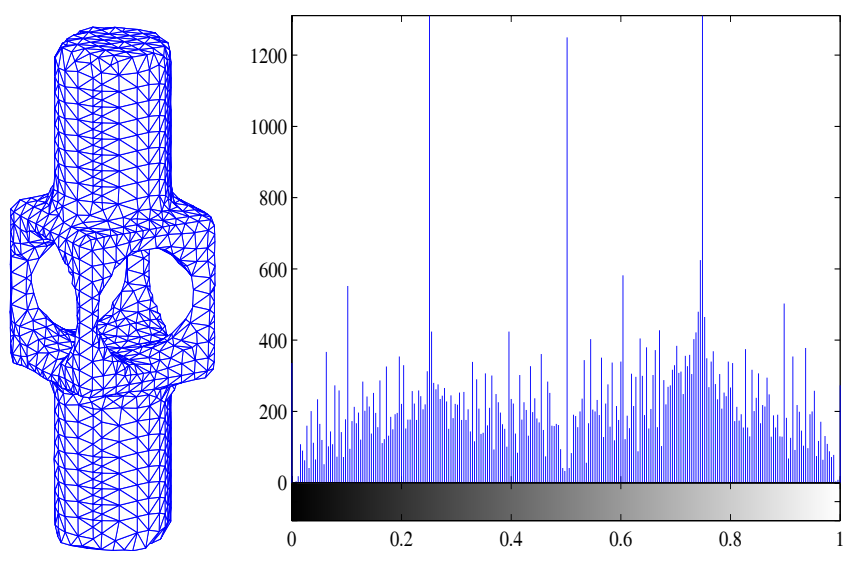

Fig. 3. 3D object and its shape spectrum.

The height function, however, has some limitations as an object signature for matching, indexing, or retrieval of arbitrary $3 \mathrm{D}$ objects. The main reason is that the height function is not rotationally invariant. To overcome these limitations, we propose a global geodesic function defined on the object surface as follows. Let $\boldsymbol{c}_{i}$ and $\boldsymbol{c}_{j}$ be two points (centroids) on a smooth manifold $\mathcal{M}$. The geodesic distance $g\left(\boldsymbol{c}_{i}, \boldsymbol{c}_{j}\right)$ between $\boldsymbol{c}_{i}$ and $\boldsymbol{c}_{j}$ is the shortest length $L(\gamma)=\int_{a}^{b}\left\|\gamma^{\prime}(t)\right\| d t$ of a smooth curve $\gamma:[a, b] \rightarrow \mathcal{M}$ such that $\gamma(a)=\boldsymbol{c}_{i}$ and $\gamma(b)=\boldsymbol{c}_{j}$. The geodesic distance may be locally viewed as the Euclidean $d_{E}\left(\boldsymbol{c}_{i}, \boldsymbol{c}_{j}\right)=\left\|\boldsymbol{c}_{i}-\boldsymbol{c}_{j}\right\|$, and is hence clearly invariant to rotation and translation.

Inspired by the geodesic-based representation for 3D topology matching proposed by Hilaga et al. [19], we define a global shape function $f: \mathcal{C} \rightarrow \mathbb{R}$ expressed in terms of a rotationally invariant (square) geodesic distance as follows

$$
f\left(\boldsymbol{c}_{i}\right)=\frac{1}{|\mathcal{C}|} \sum_{j=1}^{n} \operatorname{area}\left(T_{j}\right) g\left(\boldsymbol{c}_{i}, \boldsymbol{c}_{j}\right)^{2} .
$$

The primary motivation behind the geodesic distance is of overcoming the limitations of the Euclidean distance which by virtue of its linearity in nature cannot account for nonlinear structures in the underlying object. Unlike the Euclidean distance which is basically a straight line between two points in 3D space, the geodesic distance captures the global nonlinear structure and the intrinsic geometry of the data as illustrated in Fig. 4. This clearly shows that the Euclidean distance between two arbitrary points in a nonlinear manifold is just a straight segment connecting two points and does not reflect the nonlinear structure of the object, whereas the geodesic distance which is the shortest curve along the manifold connecting both points clearly captures the intrinsic geometry of the object.

1) Geodesic distance calculation: Given a set of centroids $\mathcal{C}=\left\{\boldsymbol{c}_{1}, \ldots, \boldsymbol{c}_{n}\right\}$ of a $3 \mathrm{D}$ object represented by a triangle mesh $\mathbb{M}$, the geodesic distance calculation is based on a similar approach used for computing the isometric feature mapping (Isomap) for multidimensional scaling [29] on nonlinear manifolds [30]. The algorithm has two main steps:

(i) Construct a neighborhood graph by connecting a given

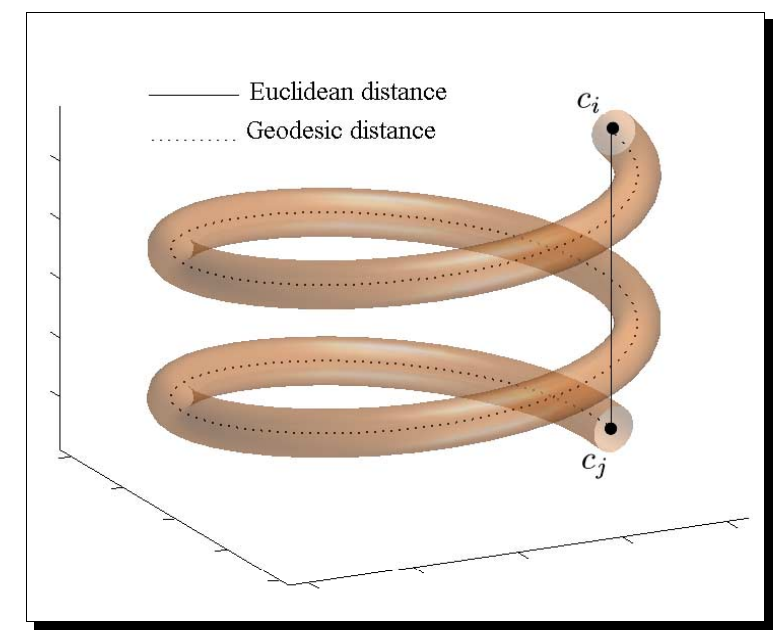

Fig. 4. Euclidean vs. geodesic distance on a nonlinear manifold.

centroid to its $k$-nearest neighbors, and link these neighboring centroids by edges with weights equal to the Euclidean distances.

(ii) Compute the geodesic distances (shortest paths) between all pairs of $n$ points in the constructed graph using Dijkstra's or Floyd's algorithm.

Note that Step (i) may be alleviated by choosing a random subset of $\mathcal{C}$ in order to reduce the computational complexity of the geodesic calculation. From Eq. (2), it is clear that a discrete form of the geodesic shape function can be written as

$$
f\left(\boldsymbol{c}_{i}\right)=\frac{(\boldsymbol{G a})_{i}}{|\mathcal{C}|}=\frac{(\boldsymbol{G a})_{i}}{\|\boldsymbol{a}\|_{1}}, \quad i=1, \ldots, n
$$

where $\boldsymbol{G}=\left(g_{i j}^{2}\right)$ is the (square) geodesic distance matrix of size $n \times n$, and $\boldsymbol{a}=\left(a_{1}, \ldots, a_{n}\right)^{T}$ is an $n \times 1$ vector of triangle areas, i.e. $a_{j}=\operatorname{are} a\left(T_{j}\right)$, and $|\mathcal{C}|=\sum_{j=1}^{n} a_{j}=\|\boldsymbol{a}\|_{1}$ is the total area. The geodesic distance matrix $\boldsymbol{G}=\left(g_{i j}^{2}\right)$ is symmetric with zeros in the diagonal, and positive off-diagonal elements. Note that the geodesic distance on triangulated surfaces may also be effectively computed using the fast marching method introduced in [31].

2) Triangle area calculation: Denote by $\left\{\boldsymbol{v}_{1}, \boldsymbol{v}_{2}, \boldsymbol{v}_{3}\right\}$ the vertices of an arbitrary triangle $T$ of a given triangle mesh M. Using Newell method, the area of the triangle $T$ can be computed as $\operatorname{area}(T)=\|\boldsymbol{N}\| / 2$, where $\boldsymbol{N}=\left(N_{1}, N_{2}, N_{3}\right)$ is the triangle normal vector with coordinates given by

$$
\begin{aligned}
& N_{1}=\sum_{i=1}^{d}\left(y_{i}-y_{\operatorname{next}(i)}\right)\left(z_{i}+z_{\operatorname{next}(i)}\right) \\
& N_{2}=\sum_{i=1}^{d}\left(z_{i}-z_{\operatorname{next}(i)}\right)\left(x_{i}+x_{\operatorname{next}(i)}\right) \\
& N_{3}=\sum_{i=1}^{d}\left(x_{i}-x_{\operatorname{next}(i)}\right)\left(y_{i}+y_{\operatorname{next}(i)}\right)
\end{aligned}
$$

and $\left(x_{i}, y_{i}, z_{i}\right)$ are the coordinates of each vertex $\boldsymbol{v}_{i}$ (with dimension $d=3$ ) of a triangle $T$. Note that $\boldsymbol{v}_{\operatorname{next}(i)}=$ $\left(x_{\text {next }(i)}, y_{\text {next }(i)}, z_{\text {next }(i)}\right)$ denotes the next vertex in the list after $\boldsymbol{v}_{i}$, taking into account that $\boldsymbol{v}_{1}$ follows the last vertex $\boldsymbol{v}_{d}$. 


\section{B. Global geodesic shape distribution}

Note that the geodesic shape function can be expressed as a geodesic shape vector $\mathcal{X}=\left\{X_{1}, \ldots, X_{n}\right\}$, where $X_{i}=f\left(\boldsymbol{c}_{i}\right)$. This vector may be viewed as a shape descriptor that may be used for 3D shape comparison.

Assume that the geodesic shape vector $\mathcal{X}$ of an object $\mathbb{M}$ is a random sample with a common (unknown) probability density function $p$. A common approach to approximate the probability density function $p$ is through the kernel density estimation which is an important data analytic tool that provides a very effective way of showing structure in a data set [32]. The kernel density estimator $\hat{p}$ is given by

$$
\hat{p}(x)=\frac{1}{n h} \sum_{i=1}^{n} K\left(\frac{x-X_{i}}{h}\right),
$$

where $x$ represents the normalized value of the geodesic shape function given by Eq. (3), $K$ is the Gaussian kernel, and $h$ is the bandwidth or window width to be estimated. A good selection rule of this bandwidth is given by

$$
\hat{h}=\left[\frac{243 R(K)}{35 \mu_{2}(K)^{2} n}\right]^{1 / 5} \hat{\sigma}
$$

where $R(K)=\int K(t)^{2} d t, \mu_{2}(K)=\int t^{2} K(t) d t$, and $\hat{\sigma}=$ $\operatorname{med}_{j}\left\{\left|X_{j}-\operatorname{med}_{i}\left\{X_{i}\right\}\right|\right\}$ is the median absolute deviation. The effect of the bandwidth parameter $h$ is illustrated in Fig. 5.

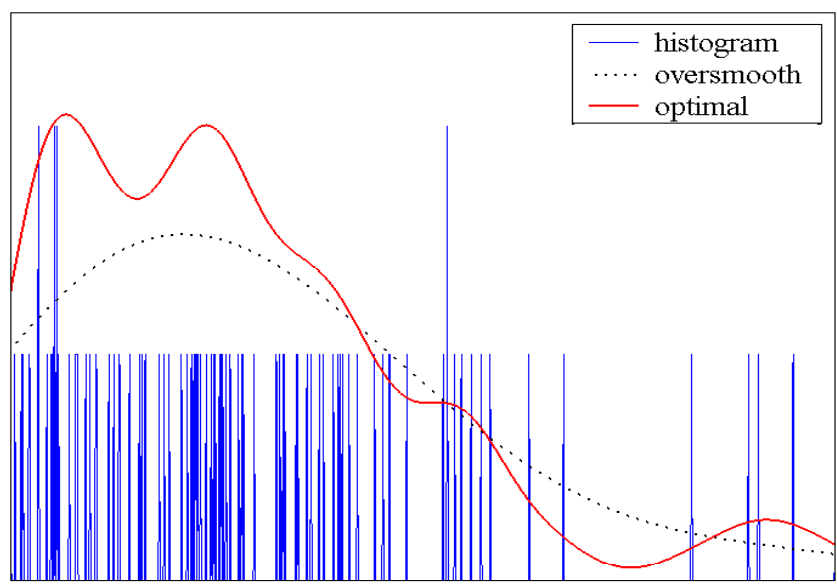

Fig. 5. Effect of the bandwidth parameter $h$. The horizontal axis represents the normalized value of the geodesic shape function, and the vertical axis represents the geodesic shape density.

The horizontal axis, of all the figures depicting the geodesic shape distributions, represents the normalized value of the geodesic shape function given by Eq. (3), whereas the vertical axis represents the geodesic shape density given by Eq. (4) with an appropriate estimated bandwidth. In other words, the geodesic shape density has a unit interval domain $[0,1]$, and a non-negative bounded interval range $[0, \mu]$ where $\mu$ is the upper bound of the density $\hat{p}$.

Hence to each 3D object represented by a triangle mesh $\mathbb{M}$, we associate a kernel density $\hat{p}$ which we will refer to as a geodesic shape distribution, and it is computed using

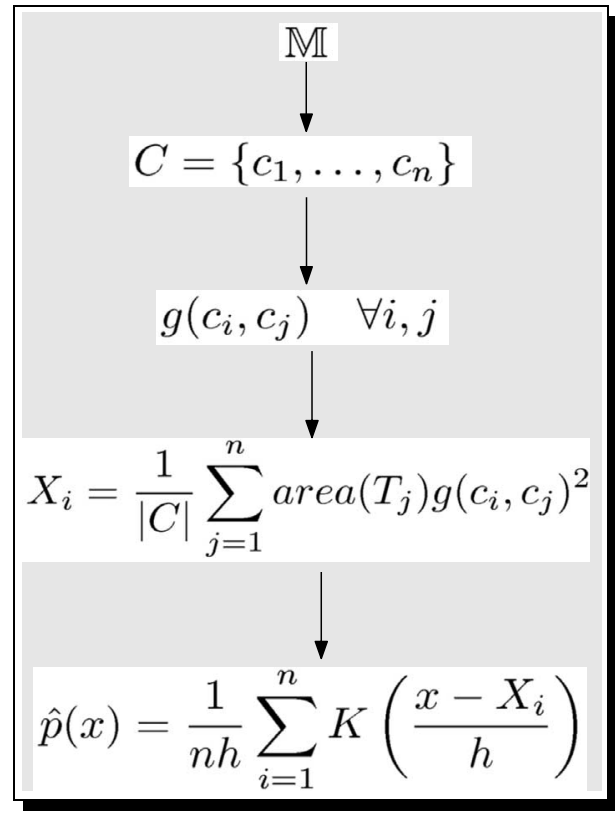

Fig. 6. Illustration of the geodesic shape distribution algorithm.

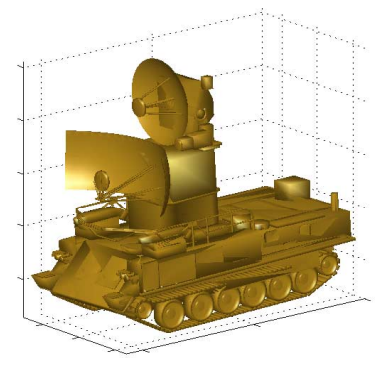

(a)

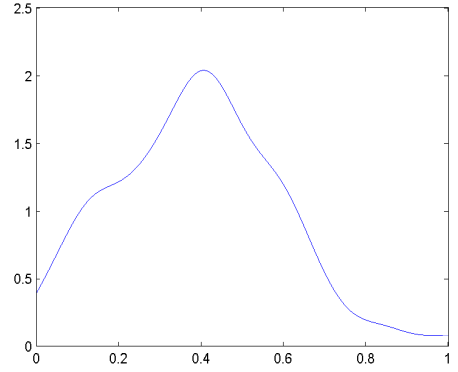

(b)
Fig. 7. (a) 3D tank model. (b) Geodesic shape distribution, where the horizontal axis represents the normalized value of the geodesic shape function, and the vertical axis represents the geodesic shape density.

the algorithm depicted in Fig. 6. This probabilistic shape descriptor represents an object information and will be used in our matching experiments. Fig. 7 depicts a 3D model of a tank and its geodesic shape distribution.

\section{Properties of geodesic shape signature}

In addition to its rotational, translational and scale invariance, the geodesic shape signature is also robust to resampling and simplification as illustrated in Fig. 8 and Fig. 9. Note that for triangulation, we use the barycentric subdivision shown in the top row of Fig. 8. This subdivision technique consists of introducing a new vertex at the center of each triangle and a new vertex at the midpoint of each edge and drawing edges from the centroid of the triangle to each of the new midpoint vertices and to the original vertices.

In order to compare two geodesic shape distributions and hence to measure the performance of the proposed scheme, we will describe in the next section an information-theoretic distance that quantifies the difference between two 3D shapes through their probabilistic shape descriptors. Fig. 10 depicts a 

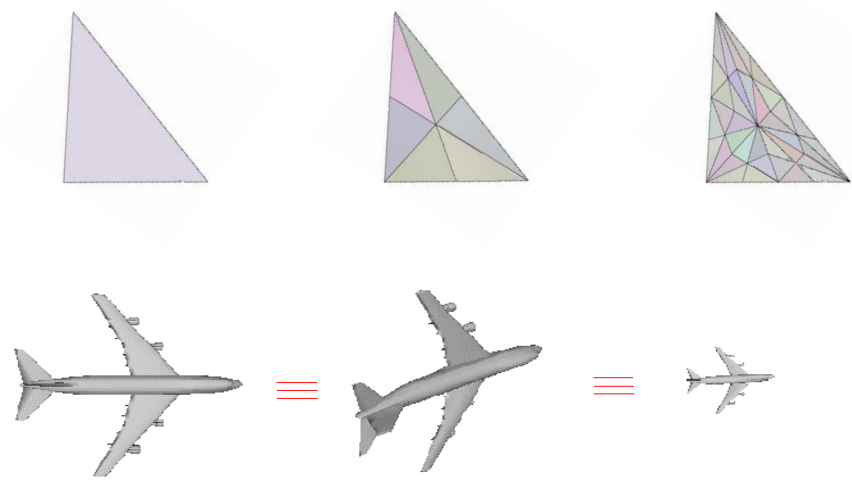

Fig. 8. Robustness and invariance.

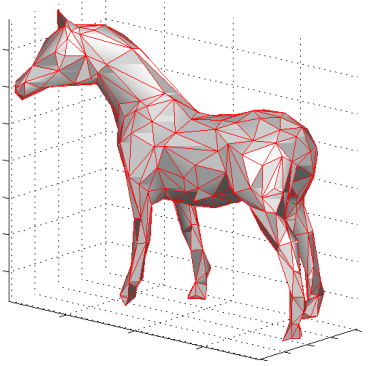

(a) $\mathbb{M}_{1}$

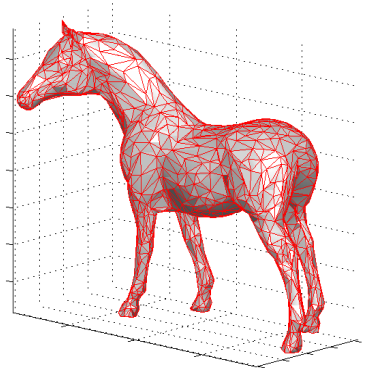

(c) $\mathbb{M}_{3}$

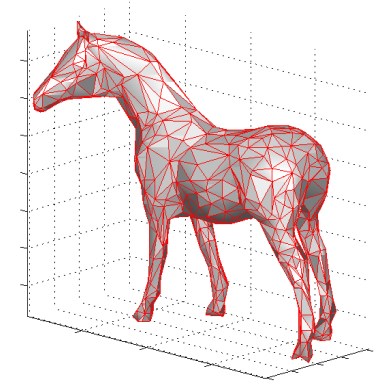

(b) $\mathbb{M}_{2}$

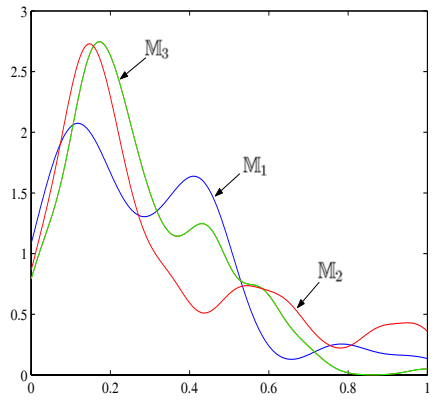

(d) geodesic shape distributions
Fig. 9. Robustness and invariance (cont.). In (d) The horizontal axis represents the normalized value of the geodesic shape function, and the vertical axis represents the geodesic shape density.

block-diagram of the proposed framework.

\section{PROBABILISTIC DISSIMILARITY}

Let $\mathbb{M}_{1}$ and $\mathbb{M}_{2}$ be two 3D objects with geodesic shape distributions $\hat{p}$ and $\hat{q}$ respectively. Information theoretic measures provide quantitative entropic divergences between two probability distributions. A common entopic dissimilarity measure is Kulback-Liebler (KL) divergence $\mathcal{K}$ defined as

$$
\mathcal{K}(\hat{p}, \hat{q})=\int \hat{p}(x) \log _{2} \frac{\hat{p}(x)}{\hat{q}(x)} d x=\mathbb{E}\left\{\log \frac{p(x)}{q(x)}\right\},
$$

where $\mathbb{E}\{\cdot\}$ denotes the expected value with respect to $p(x)$.

The KL dissimilarity measure, however, is non-symmetric, unbounded, and undefined if $\hat{p}$ is not absolutely continuous with respect to $\hat{q}[16,33]$. To overcome these limitations, we

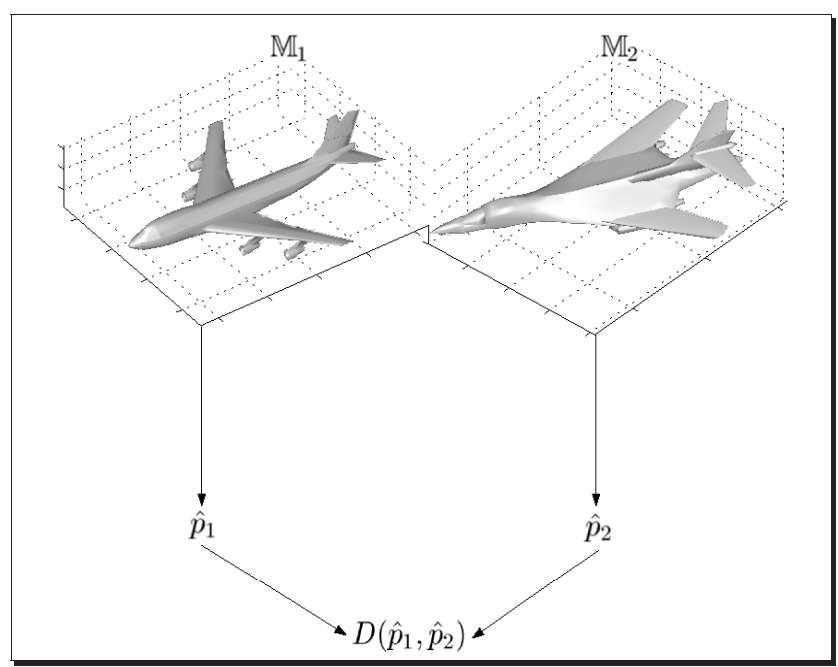

Fig. 10. Block-diagram of the proposed methodology.

use the Jensen-Shannon (JS) divergence $D$ given by

$$
\begin{aligned}
D(\hat{p}, \hat{q}) & =\frac{1}{2}\left[\mathcal{K}\left(\hat{p}, \frac{\hat{p}+\hat{q}}{2}\right)+\mathcal{K}\left(\hat{q}, \frac{\hat{p}+\hat{q}}{2}\right)\right] \\
& =H\left(\frac{\hat{p}+\hat{q}}{2}\right)-\frac{H(\hat{p})+H(\hat{q})}{2},
\end{aligned}
$$

where $H(\hat{p})=-\int \hat{p}(x) \log _{2} \hat{p}(x) d x$ is the differential entropy, which corresponds to Shannon's entropy in the discrete domain. Shannon's entropy is a measure of uncertainty, dispersion, and randomness. The maximum uncertainty is achieved by the uniform distribution, so we can think of the entropy as a measure of uniformity of a probability distribution. Hence, when uncertainty is higher it becomes more difficult to predict the outcome of a draw from a probability distribution.

The JS divergence is a statistical distance that is very useful in quantifying differences between probability distributions or densities. In other words, this dissimilarity measure quantifies differences in shape between two arbitrary objects. Unlike the Kullback-Leibler divergence, the JS divergence has the advantage of being symmetric, always defined, and generalizable to any arbitrary number of probability distributions, with a possibility of assigning weights to these distributions [12]. Fig. 11 shows a three-dimensional graph and a contour plot of the JS divergence between two discrete Bernoulli distributions. The following result establishes the convexity of the JS divergence [14].

Proposition 1: The Jensen-Shannon divergence $D(\hat{p}, \hat{q})$ is a convex function of $\hat{p}$ and $\hat{q}$.

In addition to its convexity property, the JS divergence is shown to be an adapted measure of disparity among probability distributions. Using the theory of majorization, it can be shown that the JS divergence is bounded, and its upper bound is achievable [14].

Proposition 2: The Jensen-Shannon divergence between two geodesic shape distributions $\hat{p}$ and $\hat{q}$ is upper bounded

$$
D(\hat{p}, \hat{q}) \leq \log _{2}(2)=1 .
$$




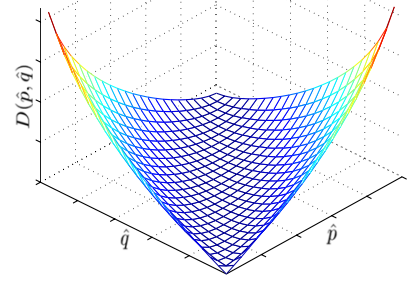

(a)

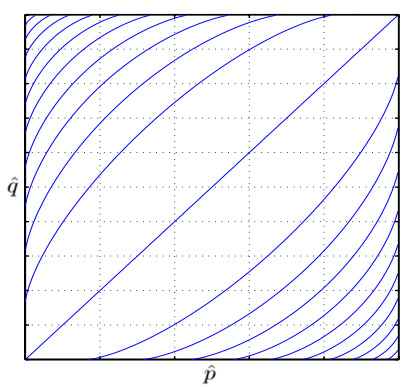

(b)
Fig. 11. (a) 3D plot and (b) contour plot of the Jensen-Shannon divergence.

\section{EXPERIMENTAL RESULTS}

Object matching experiments were performed using a database of 3D models collected online. Each model is represented as a triangle mesh which consists of a pre-selected number of triangles equal to 1000 in all the experiments. We conducted the following five sets of experiments:

\section{A. Experiment \#1: 3D airplanes}

The first set consists of 3D airplane models as shown in Fig. 12. As can be seen, the only difference between the models $\mathbb{M}_{1}$ and $\mathbb{M}_{3}$ is the radar. Using the Jensen-Shannon divergence, we computed the pairwise dissimilarity values between the models, and we found indeed that the smallest value corresponds to the dissimilarity between $\mathbb{M}_{1}$ and $\mathbb{M}_{3}$ with a matching rate of $99 \%$.
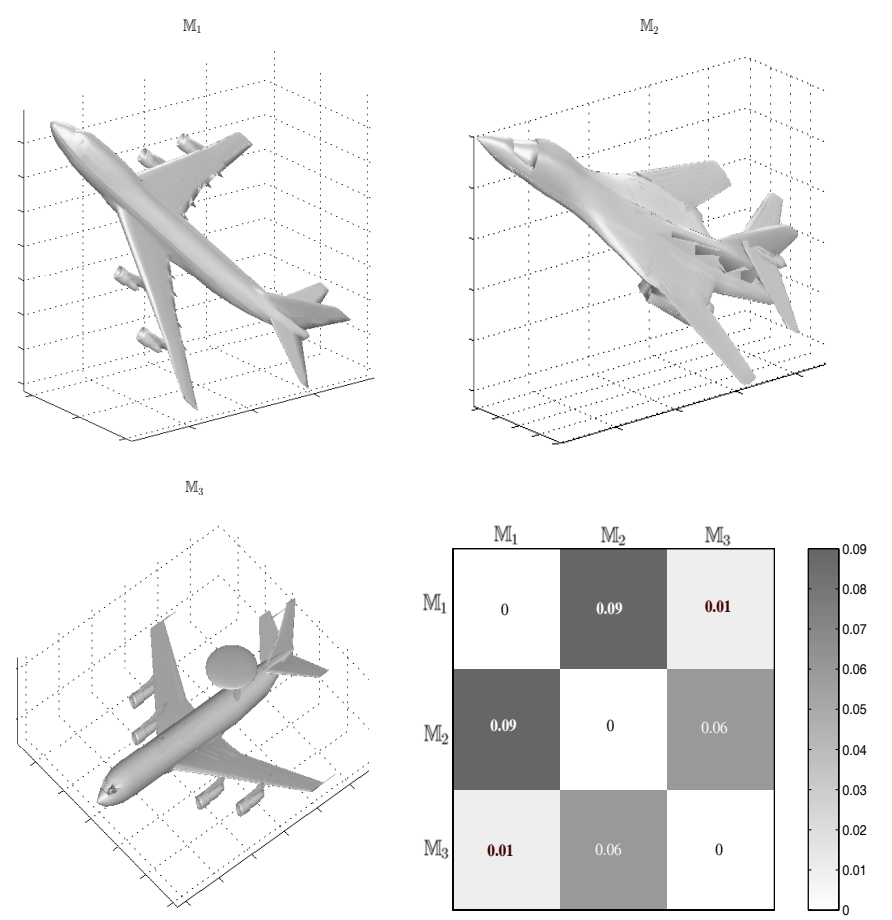

Fig. 12. First set of experiments: 3D airplanes.

\section{B. Experiment \#2: 3D tanks}

The second set consists of 3D tanks as illustrated in Fig. 13. Similar to the first experiment, we computed the pairwise dissimilarity values between the models, and we found that the smallest value corresponds to the dissimilarity between $\mathbb{M}_{1}$ and $\mathbb{M}_{3}$ with a matching rate above $98 \%$.
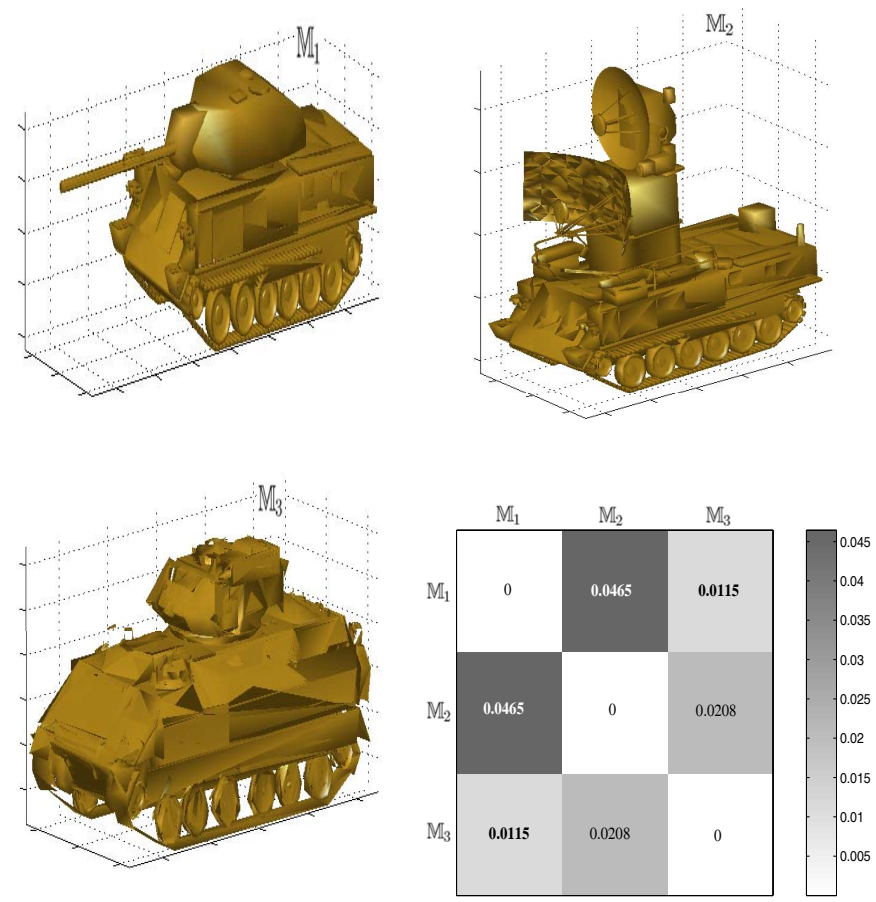

Fig. 13. Second set of experiments: 3D tanks.

\section{Experiment \#3: 3D models of animals}

The third set deals with objects that are topologically equivalent to a sphere (i.e. with genus equal to zero) as shown in Fig. 14. The numerical results using the JensenShanon dissimilarity measure are depicted in Table I where the grayscale colorbar displays the grayscale colormap of this dissimilarity matrix. This grayscale colormap ranges from white (maximum similarity) to black (maximum dissimilarity), and passes through the gray colors indicating the values of the matching algorithm. Note that the minimum dissimilarity rate is about $9 \%$, that is the matching rate is about $91 \%$.

\section{Experiment \#4: 3D models with handles}

In the fourth set of experiments, the underlying objects are topologically different from the ones considered in the third set of the experiments. Fig. 15 shows a set of objects with genus equal to one. Matching is achieved by the minimum JensenShannon distance computations as illustrated in Table II. Note that the minimum dissimilarity rate is about $2 \%$, that is the matching rate is about $98 \%$.

\section{E. Experiment \#5: 3D face matching}

An important application of the geodesic shape signature is 3D face recognition as illustrated in Fig. 16, and the dissimilarity values between these 3D face models are given in Table III. The results indicate that faces $\mathbb{M}_{3}$ and $\mathbb{M}_{5}$ are the most similar with a matching rate above $98 \%$. 

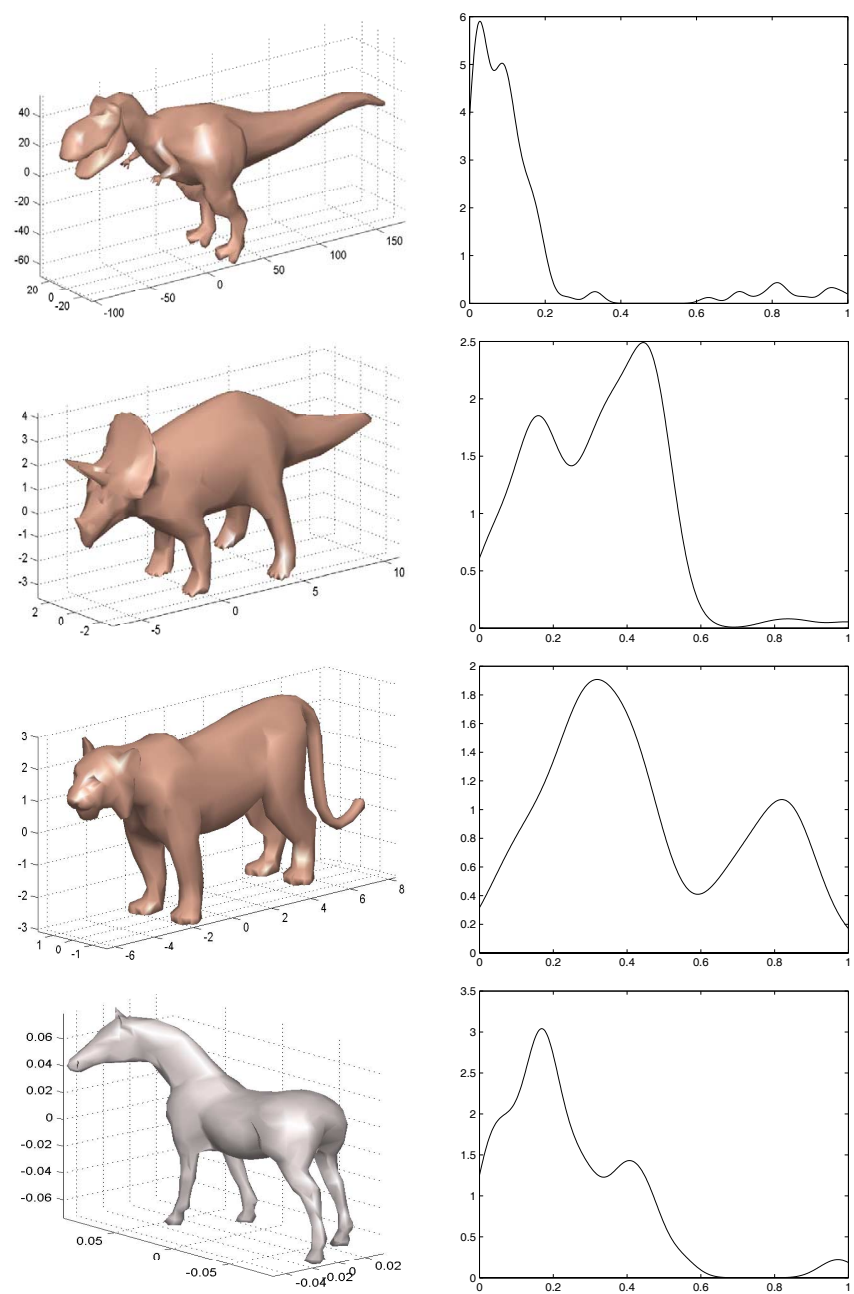

Fig. 14. Third set of experiments: 3D models and their geodesic shape distributions.

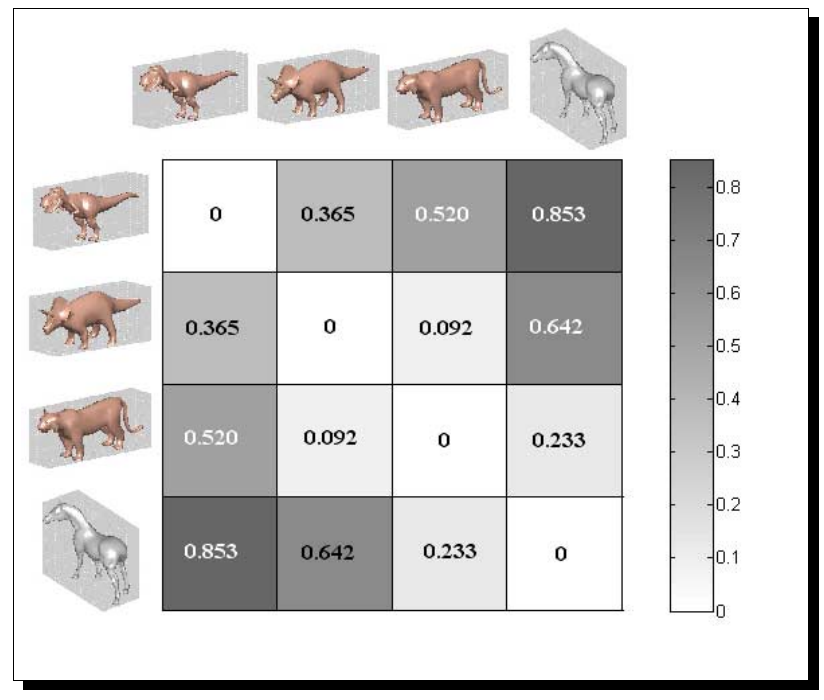

TABLE I

JENSEN-SHANNON DISSIMILARITY RESULTS FOR THE THIRD SET OF EXPERIMENTS.
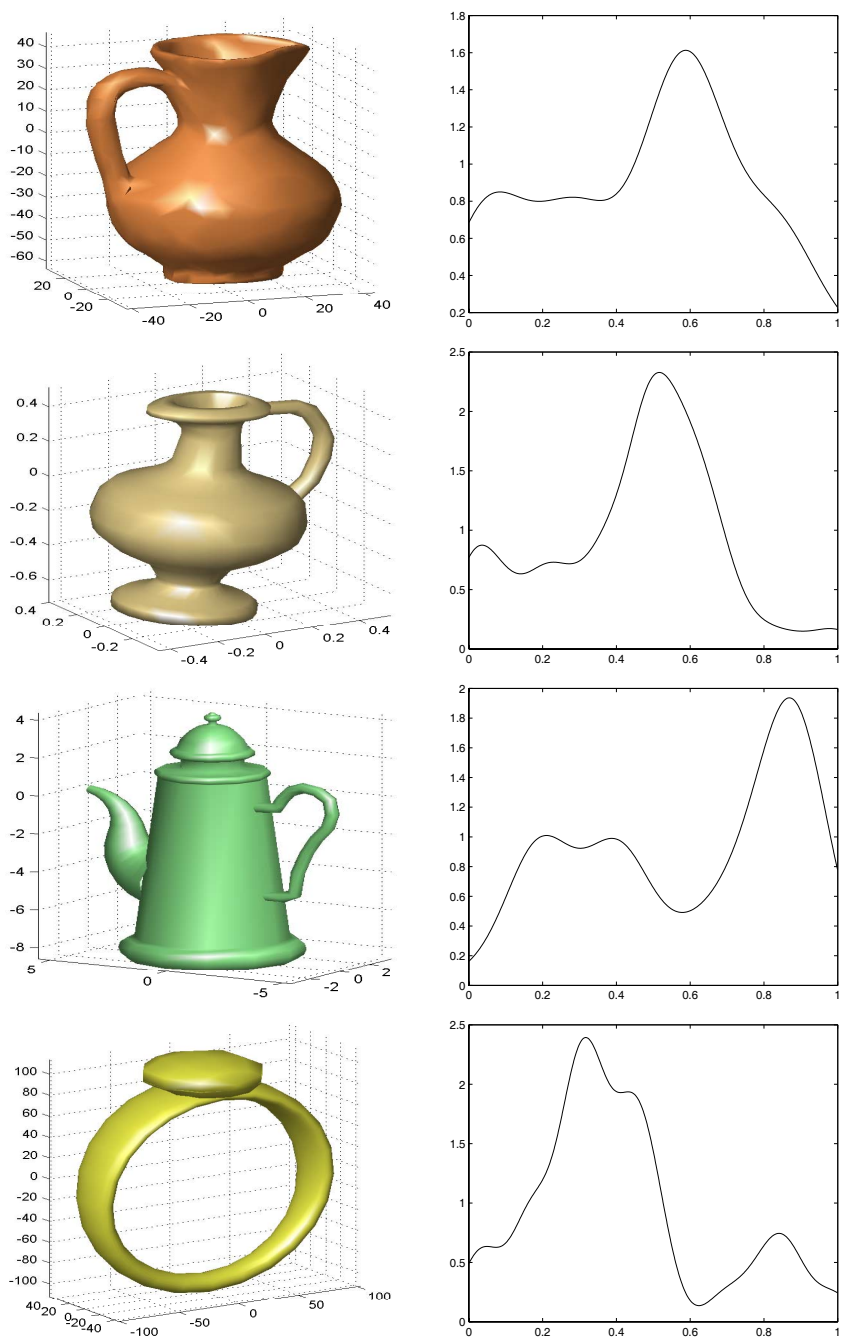

Fig. 15. Fourth set of experiments: 3D models and their geodesic shape distributions.

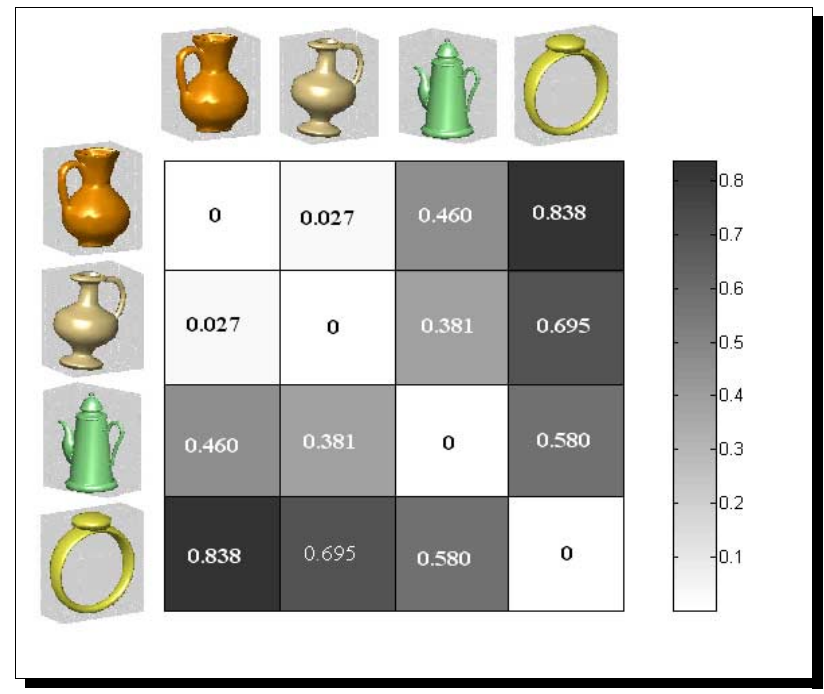

TABLE II

JENSEN-SHANNON DISSIMILARITY RESULTS FOR THE FOURTH SET OF EXPERIMENTS. 


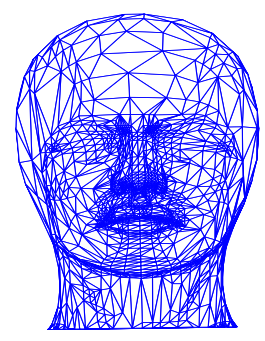

$\mathbb{M}_{1}$

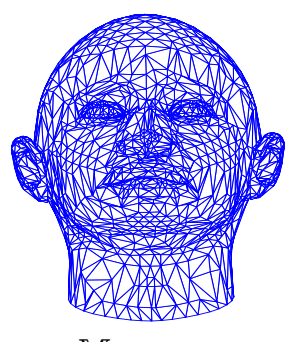

$\mathbb{M}_{3}$

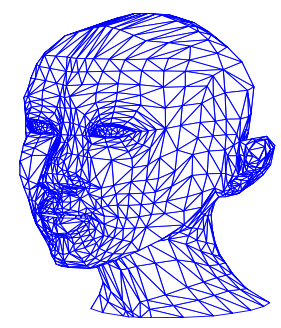

$\mathbb{M}_{5}$

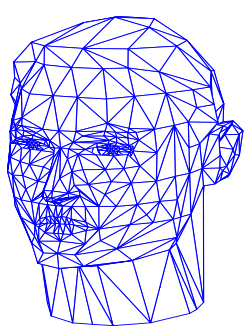

$\mathbb{M}_{2}$

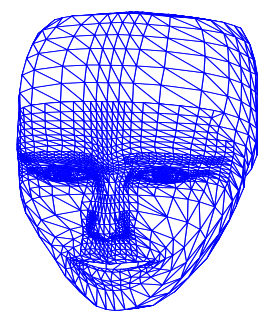

$\mathbb{M}_{4}$

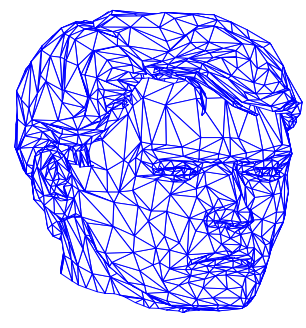

$\mathbb{M}_{6}$

Fig. 16. 3D face models.

\begin{tabular}{||l||l|l|l|l|l|l||}
\hline \multirow{2}{*}{ 3D face models } & \multicolumn{7}{|c||}{ Jensen-Shannon dissimilarity: $D\left(\mathbb{M}_{i}, \mathbb{M}_{j}\right)$} \\
\cline { 2 - 8 } & $\mathbb{M}_{1}$ & \multicolumn{1}{|c||}{$\mathbb{M}_{2}$} & \multicolumn{1}{|c||}{$\mathbb{M}_{3}$} & \multicolumn{1}{|c|}{$\mathbb{M}_{5}$} & $\mathbb{M}_{6}$ \\
\hline \hline $\mathbb{M}_{1}$ & 0 & 0.06 & 0.021 & 0.05 & 0.029 & 0.08 \\
\hline $\mathbb{M}_{2}$ & 0.06 & 0 & 0.03 & 0.08 & 0.043 & 0.025 \\
\hline $\mathbb{M}_{3}$ & 0.021 & 0.03 & 0 & 0.092 & $\mathbf{0 . 0 1 2}$ & 0.054 \\
\hline $\mathbb{M}_{4}$ & 0.05 & 0.08 & 0.092 & 0 & 0.11 & 0.097 \\
\hline $\mathbb{M}_{5}$ & 0.029 & 0.043 & $\mathbf{0 . 0 1 2}$ & 0.11 & 0 & 0.046 \\
\hline $\mathbb{M}_{6}$ & 0.08 & 0.025 & 0.054 & 0.097 & 0.045 & 0 \\
\hline
\end{tabular}

TABLE III

3D FACE MODELS: DISSIMILARITY RESULTS. THE BOLDFACE NUMBERS INDICATE THE BEST MATCHING RATE, AND ARE INDICATED TWICE IN THE TABLE DUE THE SYMMETRY OF THE DISSIMILARITY MEASURE.

\section{F. Computation complexity}

The computational complexity and memory requirement of the the geodesic shape distribution is dominated by the calculation of all pair shortest paths. Given a set of $m$ centroids on the surface, the Floyd algorithm requires $\mathcal{O}\left(\mathrm{m}^{3}\right)$ operations and stores $\mathcal{O}\left(\mathrm{m}^{2}\right)$ elements of estimated geodesic distances for straightforward implementations. Significant improvement could be achieved by using the fast marching method for computing the geodesic distance on triangular meshes. This algorithm was recently proposed in [31] with an overall time complexity of $\mathcal{O}(m \log m)$.

\section{G. Comparison to related techniques}

For each of the above five experiments, we computed the Jensen-Shannon dissimilarity measure between the objects using the shape spectrum, the D2 shape signature and the geodesic shape signature. The results obtained are shown in Table IV which clearly indicate that our proposed signature performs the best in terms of matching rate.

\begin{tabular}{||l||l|l|l|l|l||}
\hline \multirow{2}{*}{$\begin{array}{l}\text { Shape } \\
\text { Signature }\end{array}$} & \multicolumn{5}{|||}{ Jensen-Shannon dissimilarity: $D\left(\mathbb{M}_{i}, \mathbb{M}_{j}\right)$} \\
\cline { 2 - 6 } & Exp. \#1 & Exp. \#2 & Exp. \#3 & Exp. \#4 & Exp. \#5 \\
\hline \hline $\begin{array}{l}\text { shape } \\
\text { spectrum }\end{array}$ & 0.048 & 0.06 & 0.021 & 0.05 & 0.029 \\
\hline $\begin{array}{l}\text { D2 shape } \\
\text { signature }\end{array}$ & 0.06 & 0.036 & 0.03 & 0.08 & 0.043 \\
\hline $\begin{array}{l}\text { geodesic } \\
\text { signature }\end{array}$ & $\mathbf{0 . 0 1}$ & $\mathbf{0 . 0 1}$ & $\mathbf{0 . 0 1}$ & $\mathbf{0 . 0 2 9}$ & $\mathbf{0 . 0 1}$ \\
\hline
\end{tabular}

TABLE IV

COMPARISON RESULTS. THE BOLDFACE NUMBERS INDICATE THE BEST MATCHING RATE WHICH CORRESPOND TO THE GEODESIC SHAPE DISTRIBUTION.

\section{H. Future work}

In this preliminary work, we are mainly dealing with object representation and matching based on the geodesic shape signature using a small database. Our future goal is to extend the proposed signature to object retrieval which requires a relatively large database of 3D objects such as the Princeton benchmark database which would be very useful for testing and evaluation. For retrieval purposes, it would make more sense to look at the relative dissimilarity measures by plotting the precision versus recall, where

$$
\text { precision }=\frac{\text { No. relevant objects retrieved }}{\text { Total No. objects retrieved }}
$$

and

$$
\text { recall }=\frac{\text { No. relevant objects retrieved }}{\text { Total No. relevant objects in the collection }} .
$$

Also, it would be of interest to incorporate topology into the proposed methodology through Morse singularities of the global geodesic shape function. Finally we note that while the experimental results presented in this section are very promising, significant additional performance gains are still possible. For example, our current way of selecting centroids as landmarks is rather one of many possible options and by no means the best option, and a multiresolution geodesic shape distribution may also provide better key to landmarks.

\section{CONCLUSIONS}

In this paper, we proposed an new methodology for 3D object matching. The key idea is to encode a 3D shape into a 1D geodesic shape distribution. Object matching is then achieved by calculating an information-theoretic measure of dissimilarity between the probability distributions. That is, the dissimilarity computations are carried out in a low-dimensional 
space of geodesic shape distributions. The main advantages of the proposed approach are: (i) the geodesic distance captures the intrinsic geometry of the data, (ii) the approach is simple and computationally inexpensive, and (iii) the simulations results indicate the suitability of the proposed technique for object matching.

\section{ACKNOWLEDGMENTS}

The authors would like to thank the anonymous reviewers for helpful and very insightful comments. This work was supported in part by US Air Force Office of Scientific Research Grant no. F49620-98-1-0190. The first author was supported in part by NSERC Discovery Grant no. 311656, and NATEQ Grant no. 2006-NC-106879.

\section{REFERENCES}

[1] Y. Shinagawa, T.L. Kunii, and Y.L. Kergosien, "Surface coding based on Morse theory," IEEE Comp. Graph. and Appl., vol. 11 no. 5 , pp. 66-78, 1991.

[2] S. Yamany and A. Farag, "Surface signatures: an orientation independent free-form surface representation scheme for the purpose of objects registration and matching ," IEEE Trans. Pattern Analysis and Machine intelligence, vol. 24, no. 8, pp. 1105-1120, Aug. 2002.

[3] Y. Shinagawa and T.L. Kunii, "Constructing a Reeb graph automatically from cross sections," IEEE Comp. Graph. and Appl., vol. 11 no. 6 , pp. 44-51, 1991.

[4] A.T. Fomenco and T.L. Kunii, Topological modeling for visualization, Springer-Verlag Tokyo, 1997.

[5] A. Ben Hamza and H. Krim, "A topological skeleton of illuminated manifolds," Proc. IEEE Int. Conf. Image Processing, September 2003.

[6] J.J. Koenderink, Solid Shape, MIT Press, Cambridge, MA, 1990.

[7] C. Lu, Y. Cao, and D. Mumford, "Surface evolution under curvature flows," J. Visual Communication and Image Representation, vol. 13, no. $1 / 2$, pp. $65-81,2002$.

[8] J.W. Bruce and P.J. Giblin, Curves and singularities, Cambridge University Press, second edition, 1992.

[9] R. Osada, T. Funkhouser, B. Chazelle, and D. Dobkin, "Shape distributions," ACM Trans. on Graphics, vol. 21, no. 4, pp. 807-832, 2002.

[10] S. Kullback and R. Liebler, "On information and sufficiency," Ann. Math. Statist., vol. 22, pp. 79-86, 1951.

[11] R. Stoica, J. Zerubia, and J.M. Francos, "Image retrieval and indexing: A hierarchical approach in computing the distance between textured images," IEEE Int. Conf. on Image Processing, Chicago, 1998.

[12] J. Lin, "Divergence measures based on the Shannon entropy," IEEE Trans. Information Theory, vol. 37, no. 1, pp. 145-151, 1991.

[13] A.O. Hero, B. Ma, O. Michel and J. Gorman,"Applications of entropic spanning graphs," IEEE Sig. Proc. Magazine, vol. 19, pp. 85-95, 2002.

[14] Y. He, A. Ben Hamza, and H. Krim, "A generalized divergence measure for robust image registration," IEEE Trans. Sig. Processing, vol. 51, no. 5, pp. 1211-1220, May 2003.

[15] A. Ben Hamza and H. Krim, "Image registration and segmentation by maximizing Jensen-Renyi divergence," Lecture Notes in Computer Science, vol. 2683, pp. 147-163, 2003.

[16] J.F. Gomez, J. Martinez, A.M. Robles, and R. Roman, "An analysis of edge detection by using the Jensen-Shannon divergence," J. Mathematical Imaging and Vision, vol. 13, no. 1, pp. 35-56, August 2000.

[17] R. Roman, P. Bernaola, and J.L. Oliver, "Sequence compositional complexity of DNA through an entropic segmentation method," Physical Review Letters, vol. 80, no. 6, pp. 1344-1347, February 1998

[18] A. Ben Hamza and H. Krim, "Geodesic object representation and recognition," Lecture Notes in Computer Science, vol. 2886, pp. 378$387,2003$.

[19] M. Hilaga, Y. Shinagawa, T. Kohmura, and T.L. Kunii, "Topology matching for fully automatic similarity estimation of 3D shapes," Proc. SIGGRAPH, pp. 203-212, August 2001.

[20] J. MacQueen, "Some methods for classification and analysis of multivariate observations," Proc. 5th Berkeley Symp. Math. Stat. and Prob., pp. 281-297, 1967.

[21] J. Milnor, Morse theory, Princeton University Press, Princeton, NJ 1963.

[22] R.S. Palais and C. Terng, Critical point theory and submanifold geometry, Lecture Notes in Mathematics, vol. 1353, 1988.
[23] J. Damon, "Local Morse theory for solutions to the heat equation and Gaussian blurring," J. Diff. Equations, vol. 115, pp. 368-401, 1995.

[24] A.H. Wallace, Differential topology: first steps, W.A. Benjamin, Inc., 1968.

[25] V. Guillemin and A. Pollack, Differential topology, Prentice-Hall, Inc., Englewood Cliffs, NJ 1974.

[26] L.C. Kinsey, Topology of surfaces, Springer-Verlag, 1993.

[27] M. do Carmo, Differential geometry of curves and surfaces, PrenticeHall, New Jersey, 1976

[28] C. Dorai and A. Jain, "Shape spectrum based view grouping and matching of 3D free-form objects," IEEE Trans. Pattern Analysis and Machine intelligence, vol. 19, no. 10, pp. 1139-1145, Oct. 1997.

[29] T.F. Cox and M.A. Cox, Mulridimensional scaling, second edition, Monographs on Statistics and Applied Probability, vol. 88, 2001.

[30] J.B. Tenenbaum, V. de Silva, and J.C. Langford, "A global geometric framework for nonlinear dimensionality reduction," Science, vol. 290, pp. 2319-2323, December 2000.

[31] R. Kimmel and J.A. Sethian, "Computing geodesic paths on manifolds," Proc. Natl. Acad. sci., vol. 95, pp. 8431-8435, July 1998.

[32] M.P. Wand and M.C. Jones, Kernel Smoothing, Chapman and Hall, London, 1995.

[33] G. Gallager, Information theory and reliable communications, John Willey Sons, 1968.

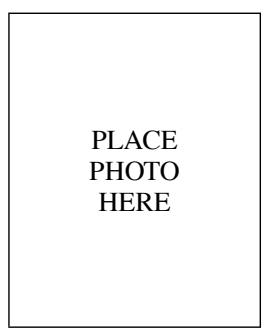

A. Ben Hamza received his Ph.D. degree in electrical engineering from North Carolina State University in 2003 , where he worked on computational imaging, 3D object recognition and information theory. He is currently an Assistant Professor in the Concordia Institute for Information Systems Engineering (CIISE) at Concordia University, Montreal, Quebec. Prior to joining CIISE, he was a postdoctoral research associate at Duke University in North Carolina, affiliated with both the Department of Electrical and Computer Engineering and the Fitzpatrick Center for Photonics and Communications Systems. His research interests include 3D graphics, computational imaging and vision, informationtheoretic measures, and human tracking using pyroelectric infrared sensors.

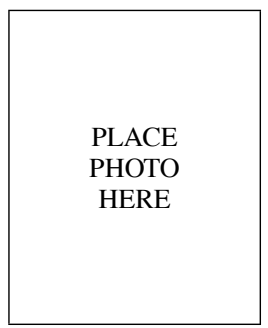

Hamid Krim received all of his degrees in electrical engineering. As a Member of Technical Staff at AT\&T Bell Labs, he has worked in the areas of telephony and digital communication systems/subsystems. Following an NSF postdoctoral fellowship at Foreign Centers of Excellence, LSS/University of Orsay, Paris, France, he became a Research Scientist at the Laboratory for Information and Decision Systems, Massachusetts Institute of Technology, Cambridge, performing and supervising research. He is currently a Professor in the ECE Department at North Carolina State University, Raleigh, leading the Vision, Information and Statistical Signal Theories and Applications group, whose research interests are in statistical signal and image analysis and mathematical modeling with a keen emphasis on applied problems. He served as Associate Editor for IEEE Transactions on Signal Processing. 